\title{
Applications of Ant Colony Optimization in Control Systems, Robotics and Vision
}

\author{
Himanshu \\ Jahagirdar \\ V.E.S.I.T. Mumbai
}

\author{
Vilas Dhuri \\ V.E.S.I.T. Mumbai
}

\author{
Pratik Bobade \\ V.I.T. Mumbai
}

\author{
Rajani Mangala \\ V.E.S.I.T. Mumbai
}

\begin{abstract}
Swarm Intelligence is driving research in multi-agent system based robotic and mobile control applications. A swarm optimization algorithm- Ant Colony Optimization (ACO) provides a stochastic 'shortest path' approach inspired by ant colonies to obtain global solution in an optimization problem. This paper reviews the impact of ACO in robotics, computer vision, control systems and sensor networks for autonomous systems. The performance of ACO as a metaheuristic or general optimization is discussed with respect to its consequences on parameters like energy efficiency, time for convergence, route selection, etc.
\end{abstract}

\section{Keywords}

Ant Colony Optimization, Robotics, Mobile Robots

\section{INTRODUCTION}

Ants are capable of finding paths from food to their nest by laying down pheromone trails and exploiting them to find a better optimized shortest path. Initially the ants wander randomly in search of food, and upon encountering the food the ants return to its nest leaving behind it trails of pheromones [1]. Other ants who come across this trail might not wander around randomly and eventually all the ants run along the trails thus making round trips from the food source to their nest. It's not necessary to have a single correct path to the food; the longer the distance from the nest to the food, the higher the rate of pheromone evaporation. Thus, the density of pheromones on longer path decreases over a period of time. On the other hand, the density of pheromones on shorter paths increases and so more ants follow the shortest available distance from nest to the food thus optimizing the distance and increasing the food collecting efficiency.

Ant Colony Optimization (ACO) in computational applications has been derived by mimicking the behavior of "simulated ants" to find a more efficient way to tackle various computational problems. An example of application of ACO is in Travelling Salesman Problem (TSP). In TSP using ACO the "simulated ants" are the agents which move from city to city guided by artificial pheromones trails and previously available heuristic information

The main idea is that the agents i.e. "simulated ants" homologous search for the best solution for TSP cooperating with the pheromone-directed communication. Each agent has its own solution for the problem which is in compliance with the previous experiences and heuristic information [3]. The agents prefer the cities which are closer and are connected with high density of pheromones trails. Each agent has a limited amount of memory in the form of pheromone deposited by ants on TSP edges. This information is used to construct a feasible solution. After trail around cities is constructed, the pheromones are updated in a way that has the shortest route between the cities and a final solution is drafted satisfying various set parameters.

ACO is a population based probabilistic algorithm used to solve computational problems majorly involving path reduction and optimization. This paper is a brief overview of the applications of ACO in autonomous systems, robotics, control and wireless sensor networks. We will discuss the viability of this algorithm as compared to others and its performance with respect to the discussed applications.

\section{WORKFLOW}

The ACO algorithm performs an iterative loop containing two basic procedures:

i) Modification/construction of solutions to the problems by the ant colony.

ii) Procedure to update pheromone trails

The construction/modification is performed in a probabilistic and stochastic way. The updates in the pheromone trail are implemented as a function that depends on the rate of pheromone evaporation and quality of the produced solution [35]. Following are the steps in ACO:

a) Initialization: The pheromone trail is initialized in the first step.

b) Iterations and pheromone update: In every iteration, each ant constructs a complete solution to the problem according to probabilistic state transition rule. This rule depends on the state of pheromone. When all ants generate a solution, a global pheromone updating rule is applied in two phases:

i) Evaporation phase: Fraction of pheromone evaporated.

ii) Reinforcement Phase: Ant deposits amount of pheromone proportional to fitness of solution

c) Termination: If the terminating condition is met, it prints the best solution from the iterations performed and terminates. If the terminating condition is not met,the ants are initialized again based on current best solution and the steps are repeated.

\section{APPLICATIONS AND PERFORMANCE}

\subsection{Motion Planning in Mobile Robots}

Path planning is necessary for mobile robots (MR), which can traverse terrains or places that are generally inaccessible to humans. ACO was primarily used for shortest path detection, for example to solve the TSP or MR motion planning. A good example of this is demonstrated in [9], where ACO is used for path routing and obstacle avoidance, which is shown through the simulation of increase in pheromone population around best solution through multiple iterations.

In [6], an ACO-MH (meta-heuristic) based algorithm called SACOmd (d for distance, $m$ for memory) was introduced for routing MR through a novel memory feature that reduced stagnation or redundancy and also included a fuzzy cost function 
that worked on both best route and best effort problems. Researchers improved the overall optimization of features set in the fuzzy rules table which determines the fuzzy logic [8]. The proposed system in [8] was specifically designed to work in a dynamic environment and was compared to a few other algorithms like genetic algorithm (GA), purely fuzzy system, basic ACO, etc. It was observed to give a reasonable if not least path cost along with the best optimization performance.

ACO has been modified multiple times in recent research to improve important parameters as discussed below. In [10], ACO was compared with ACO-PD (pheromone diffusion) and ACOPDG (geometric optimization) which differ in their usage of artificial potential field (APF) and geometric optimization are added onto the global optimization (bionic) popular with ACO. Results from multiple experiments show that the risk of premature (local) convergence is avoided, ACO-PDG results in the least cost of the three and number of iterations are significantly reduced which improves the rate of convergence. In [11], another hybrid algorithm was introduced that divided ACO into two phases- prophase (improved APF factors lead to directional movement of ants) and anaphase (cancelling prophase parameters and updating pheromones) resulting in lower path cost and higher convergence rate. Among other concurrent research, in 2017, ACO was integrated with Particle Swarm Optimization (PSO) algorithm for robot motion planning [15]. PSO is responsible for the initial distribution of pheromones in the search space which saves a lot of convergence time for ACO.

Path planning for Autonomous Underwater Vehicles (AUVs) to avoid static and energetic obstacles was presented in 2012 in [37]. Five evolutionary algorithms including ACO computed the solution to the nonlinear optimal control problem (NOCP) of the 3D path planning using six dimensional coupled equations controlled using DC motors. Particle Swarm optimization algorithm was however found to outperform ACO and deliver the highest energy efficiency. In 2018 [38], researchers introduced an additional alarm pheromone in AUVs, which prevents evaluation in areas already computed as infeasible, hence making the proposed algorithm very effective and robust. This improved algorithm gave highly accurate and stable (performs under different scale or grid dimensions) with experimental results as compared to pre-existing algorithms. Future research was proposed to implement the same in dynamic operating environment.

Unmanned Aerial Vehicles (UAVs) require trajectory planning as well, with extensive research on models to develop a good path plan. In [39], researchers used the discrete optimization feature of ACO to divide the search area into grids, consider yaw angle constraint and compute path probabilities and feasibilities using iterations of virtual ants. This research was simulated for defense purposes of locating targets and designing the shortest route. However, since the complexity of various points in the grids is high, processing power required is large. In [40], an interesting approach by researchers proposed a parallelized ACO implemented in CUDA over a GPU to reduce processing time significantly. This method tries to solve the TSP by making use of the hundreds of cores in the GPU and initializing the ants as thread blocks that can evaluate themselves parallelly. Future research was proposed to identify and handle obstacles and other real-time challenges. A certain research in this respect was done in [41], for coordinated trajectory planning in multi-UAV systems. Key features included estimated time of arrival to target (ETA), threat modelling, minimum and maximum pheromone trails to enhance performance, point pheromones for collision avoidance and determination of path and flight velocity to tackle simultaneous arrivals. In 2018 [42], while ignoring parameters like energy consumed, researchers developed ACO as a minimum time search (MTS) problem. This ACO-MTS algorithm speeds up convergence and compute high level trajectories, while pheromone trail improves the solutions in the order of few seconds. When compared with other optimization heuristics, ACO-MTS proved to require less computational time and provide a better solution. Future research involves generalizing the algorithm for all types of UAVs and develop hybrid versions of ACO-MTS for enhanced performance.

\subsection{Vehicle Routing Problem and Autonomous Vehicles}

Vehicle routing problem is a derivative of combinatorial optimization, a popular N-P problem. In a popular research done by J. E. Bell [24], ant colonies were used to emulate routes to the delivery locations, with high success in smaller problems (C1) but inefficient results with larger problems(C3,C4). A fractional approach to compute candidate list size was used to yield better results. Yu and Yang [25] made significant improvements to ACO by implementing a mutation operation inspired by its effectiveness in Genetic Algorithm and a weightbased method to update pheromones, globally as well as locally, with computational time observed for all 14 combinatorial problems(C1-C14). These researchers further developed a multiple-day time window based IACO [26] (improved) from planning period to delivery period. This IACO applied two crossover operations to minimize the probability of a local optimum and for better utilization of search space, which were then compared to the $\mathrm{C} 1-\mathrm{C} 14$ benchmarks. While most of this research has been bound to static search spaces, an enhanced ACO fused with K-means and crossover operations was implemented to evaluate vehicle routing problem in a dynamic environment [27]. While this method was observed to be slower in convergence to the rest, further research has been recommended.

A Vehicle Guidance System (VGS) was proposed as a part of Intelligent Transport System in 2010 [28]. ACO was used to calculate real-time optimum route through simulations of multiagent initializations in a mapped search space. Autonomous Intersection Management (AIM) [29] has been tackled using a combinatorial problem approach controlled by ACO, as seen in the simulations performed in [29]. While efficient for low traffic, this method gave poor performance in high traffic conditions. In [31], ACO was used in a fuzzy controller for autonomous parking application in networked vehicles by researchers who developed multi-colony parallel optimization models in MATLAB. Traffic management via Internet of Vehicles (IoV) [32] was implemented in the city of Vellore, India. Street maps were scaled to smaller segments and ACO along with a fuzzy traffic intensity cost function were used to calculate optimum path. This method proved to reduce travel time significantly after comparison with Dijkstra's, Prim's and Kruskal's algorithms.

\subsection{Computer Vision and Image Processing}

Image Edge Detection (IDE) is one of the most important stages in computer vision and image analysis routines. IDE deals with coercion of edges of a digital image by identifying points of discontinuities. IDE forms the basis of face recognition, image analysis, computer and machine vision [4]. As proposed in [5],

ACO based edge detection of images is carried out by constructing a pheromone matrix wherein each entry in the matrix represents the edge information at each pixel in the matrix. In the construction process at the end of n-th 
construction step an ant is randomly selected out of the total ants and this ant will move along the image. The ant movement is coordinated using the transition probability depending on the node pheromone value, the neighboring nodes as well as the node heuristic information. The construction process is followed by update and decision process. The end result of this proposed algorithm is compared with Nezamabadi-Pour et al.'s popular edge detection method [7]. It was concluded that in terms of visual quality of extracted edges the proposed ACO-IDE algorithm always outperformed the Nezamabadi-Pour et al.'s edge detection method.

In [4] Anna Veronica Baterina and Carlos Oppus proposed an improved ACO algorithm for IDE which is then compared with ant system (AS)[1],[13],[14]. Approved array of test images were used in this experiment and the results were compared with the outcomes of AS. The results showed that the proposed method ACS algorithm produced can extract far better edges than AS. Further, in [16], an ACO mechanism to compensate broken edges and to tackle the issue of redundant searches was presented. The ant agents are split into various groups with various labels to reduce redundant search in the same region. The labeled ants find each other with the help of the provided four redundancy-reducing policies:

\section{1) Meeting face to face \\ 2) Touching Tracks \\ 3) Adaptive tabu-lists \\ 4) Track density measurement.}

To tackle broken edge detection, compensable search techniques to select suitable path with two factors were proposed viz. Maximal neighboring difference and Maximal connective similarity [16]. The results of proposed algorithm concluded that the four redundancy-reducing policies mentioned above extracted fewer fake edges than standard ACO. Also, this policy shortened the ants search path and reduced computational complexities.

Road detection is an important element of autonomous driving. In [33], depending on the quality of segmented image, fewer or larger number of agents are required to obtain good road boundaries. Newer features have been added to ACO to make it more effective- an attraction point to polarize random movement of ants, adjusting tuning parameters for every image and a dynamic parameter changing ability.

Efficient feature selection of images for classification and recognition is important as it reduces processing time by selecting limited parameters that can deliver quality performance. In [34], by using ACO, a graphical image of $n$ features and $\mathrm{O}(\mathrm{n} 2)$ was traversed and completed in only $\mathrm{O}(2 \mathrm{n})$ arcs.

Moving object tracking through video images is an integral problem in machine vision or human computer interaction. A particle filter based on Monte Carlo method based on Bayesian filtering was improved using ACO in [36]. A particle filter ensures that with every new frame/iteration, only those sequences with heavy weights will survive. ACO interprets the particles as evaluations of the ant. This reduces degeneracy problem while also reducing computational time. This method was tested in a human tracking video sequence both in an indoor and outdoor environment [36]. ACO based particle filter was found to require the least number of samples as compared to a conventional filter.

\subsection{Control System}

Proportional-integral-devices (PID) controllers in control systems are used to regulate time domain processes. In [17], the approach of designing PID controllers as a multi-objective optimization problem while taking in account ACO is discussed. The design of PID controllers is carried out in a way that improves dynamic response and eliminates steady state error. The main traits of ACO considered here are as follows:

\section{1) Ants coexist in colonies}

2) They communicate with the deposition of pheromones

3) They use sequence of local moves to find shortest path

4) They apply a stochastic decision policy using local information

The PID controller was tuned and compared with ZieglerNichols method [18], genetic algorithm method (GAM)[19] and ant system method(ASM)[1],[13],[14]. The multi-objective technique using ACO in [17] searches for optimal parameter tuning for PID controllers and guarantees better performance in comparison with the above mentioned single-objective algorithm techniques. In the proposed technique the convergence rate for local searches is faster than GAM and ASM and the solutions are more condensed.

ACO also contributes in control problems providing efficient mechanisms to tackle computational converging problems. In

the introduced algorithm combines the concept of multi-agent optimization and fuzzy approximation for automated design of optimal control policies for continuous state dynamic systems.

The main issue with the original paper on ACO for optimal control [21] was that the resulting problem was transformed into a stochastic decision problem. In the proposed algorithm the system remains a deterministic decision problem due to fuzzy interpolation and the transition from node to node is not analogous to state transition. Here instead of assigning an ant to a certain vertex in time it is assigned to vertices according to some degree of fulfillment at the same time. Thus, by combining the frameworks of AS and ACS with fuzzy partitioning, the results show that the presented algorithm has quick converging rate, with respect to the parameters in [20].

\subsection{Wireless Sensor Networks}

All mobile cyber-physical systems are extremely energy-critical. Data aggregation of information from various sensors in the cyber-physical system is supported by a Wireless Sensor Network (WSN). Data aggregation aims to reduce power consumption, improve bandwidth and energy utilization in the WSN. The amount of data to be transmitted is reduced. [22] proposes an ant colony algorithm for data aggregation in wireless sensor networks with the help of constructing data aggregation tree in a wireless sensor network for a group of source nodes to send sensory data to a single sink node. This method proposes extension of routing paths which in turn increases the probability of intersection of routing paths. This extension of routing path is a feature of ant colony algorithm for data aggregation suggested in [23]. It was concluded on the basis of simulation results that the presented mechanism in [22] consumes less energy than the traditional data aggregation method such as DD and GIT method.

A new communication protocol is presented in [30] named as energy efficient ant-based routing algorithm (EEABR). This protocol maximizes the lifetime of WSN, wherein a colony of ants look for the path between sensor node and destination node 
that are shorter and more energy-efficient. EEABR works on the concept of processing the information in the network node rather than transmitting all data through the ants. This protocol implements the idea of reducing the memory of each ant to two records only, where the memory is created at each node that keeps record of each ant that was received and sent. This protocol proposes that it is possible to reduce the ants' length by approximately $700 \%$, and save on each ant hop the transmission of approximately 250 bytes, which is significantly better than other protocols and ensures saving of energy. To support these, a comparison was made with two different algorithms viz. basic ant-based routing algorithm (BABR) and EEABR. Four metrics were compared to analyze the performance - Average Energy, Minimum Energy, Standard Deviation, Energy Efficiency. EEABR outperformed convincingly in comparison to the abovementioned protocols in terms of reduction in energy consumption suggesting that this protocol is very effective in WSNs which need maximization of the network lifetime.

\section{CONCLUSION}

This paper gives a brief outline on the working of ACO algorithm, especially in a dynamic environment. A review of the applications of ACO in autonomous systems- routing in robots, computer vision, PID control systems, sensor networks, path planning for vehicles, etc. is carried out. The benefits provided by ACO over existing algorithms in each area are discussed. Comparisons between other existing methods to achieve similar tasks are addressed. The future promises extensive research in ACO and other swarm algorithms with respect to autonomous systems.

\section{REFERENCES}

[1] M. Dorigo, V. Maniezzo, A. Colorni, "Ant System: Optimization by a colony of cooperating agents", IEEE Transactions on Systems Man and Cybernetics-Part B, vol. 26, no. 1, pp. 29-41, 1996.

[2] Thomas STUTZLE" $\ddagger$ and Marco DORIGO IRIDIA, Université Libre de Bruxelles, Belgium \{t stutzle, $m$ dorigo \}

[3] Ant Colony System: A Cooperative Learning Approach to the Traveling Salesman Problem Marco Dorigo and Luca Maria Gambardella

[4] Baterina, Anna Veronica and Carlos Oppus. "Image edge detection using ant colony optimization." (2010).

[5] Jing Tian, Weiyu Yu, \& Shengli Xie. (2008). An ant colony optimization algorithm for image edge detection. 2008 IEEE Congress on Evolutionary Computation (IEEE World Congress on Computational Intelligence). doi:10.1109/cec.2008.4630880

[6] Garcia, M. A. P., Montiel, O., Castillo, O., Sepúlveda, R., \& Melin, P. (2009). Path planning for autonomous mobile robot navigation with ant colony optimization and fuzzy cost function evaluation. Applied Soft Computing, 9(3), 1102-1110. doi:10.1016/j.asoc.2009.02.014

[7] H. Nezamabadi-Pour, S. Saryazdi, and E. Rashedi, "Edge detection using ant algorithms," Soft Computing, vol. 10, pp. 623-628, May 2006.

[8] Purian, F. K., \& Sadeghian, E. (2013). Mobile robots path planning using ant colony optimization and Fuzzy Logic algorithms in unknown dynamic environments. 2013 International Conference on Control, Automation, Robotics and Embedded Systems (CARE). doi:10.1109/care.2013.6733718
[9] Rashid, R., Perumal, N., Elamvazuthi, I., Tageldeen, M. K., Khan, M. K. A. A., \& Parasuraman, S. (2016). Mobile robot path planning using Ant Colony Optimization. 2016 2nd IEEE International Symposium on Robotics and Manufacturing Automation (ROMA). doi:10.1109/roma.2016.7847836

[10] Liu, J., Yang, J., Liu, H., Tian, X., \& Gao, M. (2016). An improved ant colony algorithm for robot path planning. Soft Computing, 21(19), 5829-5839. doi:10.1007/s00500016-2161-7

[11] Guoliang Chen and Jie Liu, "Mobile Robot Path Planning Using Ant Colony Algorithm and Improved Potential FieldMethod," Computational Intelligence and Neuroscience, vol.2019,ArticleID1932812,10pages,2019.

[12] https://doi.org/10.1155/2019/1932812.

[13] M. Dorigo. (2007) Ant Colony Optimization, Scholarpedia,2(3):1461.[Online].Available:

[14] http://www.scholarpedia.org/article/Ant_colony_optimizati on.

[15] M. Dorigo, V. Maniezzo, and A. Colorni, Ant System: Optimization by a Colony of Cooperating Agents, IEEE Transactions on Systems, Man and Cybernetics - Part B, vol. 26, pp. 29-41, 1996.

[16] Xu, Q.-L., Cai, M.-M., \& Zhao, L.-H. (2017). The robot path planning based on ant colony and particle swarm fusion algorithm. 2017 Chinese Automation Congress (CAC). doi:10.1109/cac.2017.8242802

[17] Lu, D.-S., \& Chen, C.-C. (2008). Edge detection improvement by ant colony optimization. Pattern Recognition Letters, 29(4), 416-425. doi:10.1016/j.patrec.2007.10.021

[18] Ibtissem Chiha, Noureddine Liouane, and Pierre Borne, "Tuning PID Controller Using Multiobjective Ant Colony Optimization," Applied Computational Intelligence and Soft Computing, vol. 2012, Article ID 536326, 7 pages, 2012

[19] J. G. Ziegler and N. B. Nichols, "Optimum settings for automatic controllers," Transactions of the ASME, vol. 64, pp. $759-768,1942$

[20] A. Bagis, "Determination of the PID controller parameters by modified genetic algorithm for improved performance," Journal of Information Science and Engineering, vol. 23, no. 5, pp. 1469-1480, 2007.

[21] Van Ast, J., Babuska, R., \& De Schutter, B. (2009). Fuzzy Ant

[22] Colony Optimization for optimal control. 2009 doi:10.1109/acc.2009.5160327

[23] J. M. van Ast, R. Babuska, and B. De Schutter, "Ant colony opti- " mization for optimal control," in Proceedings of the 2008 Congress on Evolutionary Computation (CEC 2008), Hong Kong, China, June 2008, pp. 2040-2046.

[24] Liao, W.-H., Kao, Y., \& Fan, C.-M. (2008). Data aggregation in wireless sensor networks using ant colony algorithm. Journal of Network and Computer Applications, 31(4), 387-401. doi:10.1016/j.jnca.2008.02.006

[25] Misra R, Mandal C. Ant-aggregation: ant colony algorithm for optimal data aggregation in wireless sensor networks. In: International Conference on Wireless and Optical 
Communications Networks, 2006

[26] Bell, J. E., \& McMullen, P. R. (2004). Ant colony optimization techniques for the vehicle routing problem.Advanced Engineering Informatics, 18(1), 41-48. doi:10.1016/j.aei.2004.07.001

[27] Yu, Bin, Zhongzhen Yang and Baozhen Yao. "An improved ant colony optimization for vehicle routing problem.” European Journal of Operational Research 196 (2009): 171-176.

[28] Yu, B., \& Yang, Z. Z. (2011). An ant colony optimization model: The period vehicle routing problem with time windows. Transportation Research Part E: Logistics and Transportation Review, 47(2), 166-181. doi:10.1016/j.tre.2010.09.010

[29] Haitao $\mathrm{Xu}, \mathrm{Pan} \mathrm{Pu}$, and Feng Duan, "Dynamic Vehicle Routing Problems with Enhanced Ant Colony Optimization," Discrete Dynamics in Nature and Society, vol. 2018, Article ID 1295485, 13 pages, 2018. https://doi.org/10.1155/2018/1295485.

[30] Kammoun, H. M., Kallel, I., Alimi, A. M., \& Casillas, J. (2010). An adaptive vehicle guidance system instigated from ant colony behavior. 2010 IEEE International ConferenceonSystems,ManandCybernetics.doi:10.1109/ics mc.2010.5642001

[31] Wu, J., Abbas-Turki, A., \& El Moudni, A. (2011). Cooperative driving: an ant colony system for autonomous intersection management. Applied Intelligence, 37(2), 207222. doi:10.1007/s10489-011-0322-z

[32] Camilo, T., Carreto, C., Silva, J. S., \& Boavida, F. (2006). An Energy-Efficient Ant-Based Routing Algorithm for Wireless Sensor Networks. Lecture Notes in Computer Science, 49-59. doi:10.1007/11839088_5

[33] Li, Jishun \& Zheng, Guoqiang \& Liang, Zhao. (2013). Optimization of an Intelligent Controller for Parallel Autonomous Parking. TELKOMNIKA : Indonesian Journal of Electrical Engineering. 11. 10.11591/telkomnika.v11i2.1404.

[34] Zheng, Guoqiang, Zhao Liang and Jishun Li. "Optimization of an Intelligent Controller for Parallel Autonomous Parking.” (2012).

[35] Arnay, R., Acosta, L., Sigut, M., \& Toledo, J. (n.d.). Applying an Ant Colony Optimization Algorithm to an Artificial Vision Problem in a Robotic Vehicle.
International Symposium on Distributed Computing and Artificial Intelligence 2008 (DCAI 2008), 490-497. doi:10.1007/978-3-540-85863-8_57

[36] Bolun Chen, Ling Chen, Yixin Chen, Efficient ant colony optimization for image feature selection, Signal Processing, Volume 93, Issue 6,2013, Pages 1566-1576, ISSN 01651684,https://doi.org/10.1016/j.sigpro.2012.10.022.

[37] Shortest Path Finding Algorithm Using Ant Colony Optimization Er. Sarbjeet Kaur. International Journal of Engineering Research \& Technology (IJERT) ISSN: 22780181 www.ijert.org IJERT Vol. 2 Issue 6, June - 2013

[38] Zhou Hao, Xuejie Zhang, Pengfei Yu, \& Haiyan Li. (2010). Video object tracing based on particle filter with ant colony optimization. 2010 2nd International Conference on Advanced Computer Control doi:10.1109/icacc.2010.5486857

[39] Aghababa, M. P. (2012). 3D path planning for underwater vehicles using five evolutionary optimization algorithms avoiding static and energetic obstacles. Applied Ocean Research, 38, 48-doi:10.1016/j.apor.2012.06.002

[40] Ma, Y.-N., Gong, Y., Xiao, C.-F., Gao, Y., \& Zhang, J. (2018). Path Planning for Autonomous Underwater Vehicles: An Ant Colony Algorithm Incorporating Alarm Pheromone. IEEETransactions on Vehicular Technology, 1-1. doi:10.1109/tvt.2018.2882130

[41] Chao Zhang, Zhen, Z., Daobo Wang, \& Meng Li. (2010). UAV path planning method based on ant colony optimization. 2010 Chinese Control and Decision Conference. doi:10.1109/ccdc.2010.5498477

[42] Cekmez, U., Ozsiginan, M., \& Sahingoz, O. K. (2014). A UAV path planning with parallel ACO algorithm on CUDA platform. 2014 International Conference on Unmanned Aircraft Systems (ICUAS). doi:10.1109/icuas.2014.6842273

[43] Duan, H., Zhang, X., Wu, J., \& Ma, G. (2009). Max-Min Adaptive Ant Colony Optimization Approach to MultiUAVs Coordinated Trajectory Replanning in Dynamic and Uncertain Environments. Journal of Bionic Engineering, 6(2), 161-173. doi:10.1016/s1672-6529(08)60113-4

[44] Perez-Carabaza, S., Besada-Portas, E., Lopez-Orozco, J. A., de la Cruz, J. M. (2018). Ant colony optimization for multi-UAV minimum time search in uncertain domains. Applied SoftComputing,62,789-806. 